\title{
Author Correction: Felodipine induces autophagy in mouse brains with pharmacokinetics amenable to repurposing
}

\author{
Farah H. Siddiqi1,2, Fiona M. Menzies', Ana Lopez¹,3, Eleanna Stamatakou ${ }^{1,2}$, Cansu Karabiyik1, \\ Rodrigo Ureshino (1) 1,3, Thomas Ricketts', Maria Jimenez-Sanchez (1) 1,9, Miguel Angel Esteban (D) 4 , \\ Liangxue Lai ${ }^{4}$, Micky D. Tortorella ${ }^{4}$, Zhiwei Luo ${ }^{4}$, Hao Liư ${ }^{4}$, Emmanouil Metzakopian ${ }^{5}$, Hugo J.R. Fernandes ${ }^{5}$, \\ Andrew Bassett (10 ${ }^{6}$, Eric Karran ${ }^{7}$, Bruce L. Miller ${ }^{8}$, Angeleen Fleming ${ }^{1,3}$ \& David C. Rubinsztein ${ }^{1,2}$
}

Correction to: Nature Communications https://doi.org/10.1038/s41467-019-09494-2, Published online 18 April 2019.

The original version of this Article omitted the following from the Acknowledgements:

The John Black Charitable Foundation and The Robert Luff Foundation.

This has now been corrected in both the PDF and HTML versions of the Article.

Published online: 04 June 2019

\begin{abstract}
(c) Open Access This article is licensed under a Creative Commons Attribution 4.0 International License, which permits use, sharing, adaptation, distribution and reproduction in any medium or format, as long as you give appropriate credit to the original author(s) and the source, provide a link to the Creative Commons license, and indicate if changes were made. The images or other third party material in this article are included in the article's Creative Commons license, unless indicated otherwise in a credit line to the material. If material is not included in the article's Creative Commons license and your intended use is not permitted by statutory regulation or exceeds the permitted use, you will need to obtain permission directly from the copyright holder. To view a copy of this license, visit http://creativecommons.org/licenses/by/4.0/.
\end{abstract}

(C) The Author(s) 2019

\footnotetext{
${ }^{1}$ Department of Medical Genetics, Cambridge Institute for Medical Research, University of Cambridge, The Keith Peters Building, Cambridge Biomedical Campus, Hills Road, Cambridge CB2 OXY, UK. ${ }^{2}$ UK Dementia Research Institute, Cambridge Institute for Medical Research, University of Cambridge, The Keith Peters Building, Cambridge Biomedical Campus, Hills Road, Cambridge CB2 OXY, UK. ${ }^{3}$ Department of Physiology, Development and Neuroscience, University of Cambridge, Downing Street, Cambridge CB2 3DY, UK. ${ }^{4}$ Guangzhou Institutes of Biomedicine and Health, Chinese Academy of Sciences, 190 Kai Yuan Avenue, Science Park, 501530 Guangzhou, China. ${ }^{5}$ UK Dementia Research Institute, Department of Clinical Neurosciences, University of Cambridge, Cambridge CB2 OAH, UK. ${ }^{6}$ Wellcome Sanger Institute, Wellcome Genome Campus, Hinxton, Cambridgeshire CB10 1SA, UK. ${ }^{7}$ AbbVie Inc., Foundational Neuroscience Center, 200 Sidney Street, Cambridge, MA 02139, USA. ${ }^{8}$ Memory and Aging Center, Department of Neurology, University of California, San Francisco, CA, USA. ${ }^{9}$ Present address: Department of Basic and Clinical Neuroscience, King's College London, Institute of Psychiatry, Psychology and Neuroscience, Maurice Wohl Clinical Neuroscience Institute, London SE5 9RX, UK. Correspondence and requests for materials should be addressed to D.C.R. (email: dcr1000@cam.ac.uk)
} 